\title{
Flexibilidade Mental na Resolução de Problemas em Indivíduos que Cumprem Pena por Homicídio Qualificado
}

\author{
Problem-Solving Mental Flexibility in Individuals \\ who were Convicted for Murder
}

\author{
Viviane Del Pino \& Blanca Susana Guevara Werlang* \\ Pontificia Universidade Católica do Rio Grande do Sul, Porto Alegre, Brasil
}

\begin{abstract}
Resumo
Este estudo teve como objetivo avaliar a flexibilidade mental na resolução de problemas em indivíduos que cumprem pena por homicídio qualificado em penitenciária de média segurança na região metropolitana de Porto Alegre. O estudo foi quantitativo de tipo transversal. A amostra $(n=60)$ foi localizada por conveniência, contou com 30 indivíduos homicidas pareados quanto a sexo, idade, escolaridade e classe social com 30 sujeitos sem antecedentes criminais. Os instrumentos utilizados foram: Teste Wisconsin de Classificação de Cartas WCST, Teste Stroop de Cores e Palavras, os subtestes Cubos, Códigos e Vocabulário do WAIS-III, a MiniInternational Psychiatric Interview e uma ficha de dados sócio-demográficos. Nas categorias do WCST e no Teste Stroop o grupo de homicidas apresentou escores inferiores ao grupo controle com diferença estatisticamente significativa $(p<0,05)$. Concluiu-se que os indivíduos que cumprem pena por homicídio qualificado apresentam uma menor flexibilidade na resolução de problemas quando comparados a sujeitos sem antecedentes criminais.

Palavras-chave: Homicídio qualificado; flexibilidade na resolução de problemas; Teste Wisconsin de Classificação de Cartas; Teste Stroop.
\end{abstract}

\begin{abstract}
This study aimed at assessing problem-solving mental flexibility in individuals who were convicted for qualified murder, and confined in a medium-security prison within the Metropolitan Area of Porto Alegre, Brazil. The quantitative cross-research study had a convenience sample $(n=60)$ including 30 homicides, paired by gender, age, schooling and social class to 30 individuals who had no criminal records. Assessment instruments used were Wisconsin Card Sorting Test; Stroop Color and Word Test, WAIS-III Block Design, DigitSymbol Coding and Vocabulary subtests, a Mini-International Psychiatric Interview and a socio-demographic datasheet. In the WCST categories and in the Stroop Test, the group of homicides showed lower scores than the control group with a statistically significant difference $(p<0,05)$. It is concluded that individuals convicted for qualified murder show lower problem-solving flexibility, than the individuals having no criminal records. Keywords: Qualified murder; problem-solving flexibility; Wisconsin Card Sorting Test; Stroop Color and Word Test.
\end{abstract}

O entendimento do comportamento homicida revestese de grande interesse para a saúde pública diante dos altos índices anuais deste fenômeno, representando uma das principais causas de morte, anos potenciais perdidos, e um indício do grau de violência mortal que atinge a sociedade. No âmbito nacional, as taxas de mortalidade por causas externas, em especial de homicídio, foram as que tiveram maior crescimento entre 1980 e 2000 (115\%). Na região sul do país, os índices de mortalidade por homicídio tiveram um incremento de $55 \%$ entre os anos extre-

\footnotetext{
* Endereço para correspondência: Faculdade de Psicologia, Programa de Pós-Graduação em Psicologia, Pontifícia Universidade Católica do Rio Grande do Sul, Av. Ipiranga, 6681, Prédio 11, 9o andar. Caixa Postal 1429. Porto Alegre, RS, 90619-900. E-mail: bwerlang@pucrs.br ; vivi.delpino@terra.com.br
}

mos desse período (Centro Latino-americano de Estudos de Violência e Saúde Jorge Careli [CLAVES], 2002).

O homicídio pode ser entendido como o resultado de uma ação humana intencional que cause ou tenha muitas probabilidades de causar a morte de outro ser humano, seja esta ação individual ou coletiva, de fato ou como ameaça, com o uso de força ou poder físico onde se inclui o descuido e a omissão (Minayo \& Souza, 1998; Pedroso, 1995; World Health Organization [WHO], 1996). Esta definição vincula a intenção com a execução do próprio ato, em que é possível avaliar os níveis de consciência e voluntariedade no planejamento e objetivação da ação homicida. Estes níveis indicam, em termos jurídico-legais, o grau de reprovação deste crime, e assim o tipo de pena aplicada ao(s) autor(es) do homicídio.

Dentre os crimes contra a vida, o Código Penal Brasileiro (CPB) denomina homicídio no art. 121 como "matar 
alguém”, qualificando-o em três dimensões: Privilegiado, quando cometido por motivo de relevante valor moral ou social, ou sob domínio de violenta emoção após injusta provocação da vítima; Culposo, quando o indivíduo realiza ação imprópria que cria certa margem de risco e perigo aos interesses de outrem (negligência, imprudência, imperícia), e Qualificado, quando o êxito letal da vítima é desejado por motivos particulares (mercenário, torpe ou fútil), com a utilização de meios e modos de execução para tal. Neste último tipo de homicídio, agregam-se circunstâncias que elevam e intensificam a reprovabilidade do crime e evidenciam a ação intencional do autor, ou seja, que o agente quis o resultado mor te ou assumiu o risco de produzi-lo. Como crime hediondo previsto em lei no Brasil, o homicídio qualificado implica em cumprimento de pena privativa de liberdade, de 12 a 30 anos de reclusão em regime fechado e em estabelecimento de segurança máxima ou média (Pedroso, 1995).

A busca da causalidade criminosa mostra-se uma questão complexa, em que um único fator não explica completamente porque alguns indivíduos têm mais comportamentos violentos que outros. A violência fatal, como no caso do homicídio, é considerada como um fenômeno multidimensional, muitas vezes resultante da ação recíproca de fatores biológicos, sociais, psicológicos, entre outros (Dornelles, 1988; Walker, 2001).

Os avanços tecnológicos têm permitido o conhecimento das estruturas cerebrais envolvidas na mediação da agressão e violência. Estudos com técnicas de neuroimagem e neuroquímicas identificam a anatomia e funcionamento das áreas cerebrais e deste modo apontam a participação de lesões morfológicas (Blake, Pincus \& Buckner, 1995; Filley, Price, Nell, Antoinette \& Morgan, 2001) e déficits funcionais (Giancola \& Zeichner, 1994; Raine, Buchsbaum \& LaCasse, 1997) de certos centros cerebrais na gênese do comportamento violento.

Uma das áreas cerebrais que têm sido repetidamente implicada na compreensão da conduta violenta é o córtex frontal (Filley et al., 2001; Foster, Hillbrand \& Silverstein, 1993; Pennington \& Ozonoff, 1996). Neuroanatomicamente, o lobo frontal subdivide-se em três áreas: motora, pré-motora e pré-frontal, sendo que através de suas múltiplas conexões se relaciona com outras áreas de associação do cérebro. Especificamente, a área pré-frontal modula as atividades do hipotálamo e do sistema límbico e seu funcionamento tem um papel crítico na organização do comportamento, na linguagem e nas ações cognitivas (Fuster, 2002; Gauer \& Guilhermano, 2001).

Estas funções cognitivas implicam no desempenho do lobo pré-frontal em termos do controle antecipado da ação, da escolha dos objetivos a serem alcançados, do planejamento, da seleção da resposta mais adequada e da inibição de outras, da atenção no acompanhamento enquanto a ação se desenrola e da verificação do resultado (Gil, 2002; Oliveira-Souza, Ignácio, Cunha, Oliveira \& Moll, 2001).

Estes variados processos cognitivos complexos contribuem para a formação do termo "funções executivas" e envolvem a integração temporal das ações com um fim direcionado, sendo esta integração exercida pela memória de trabalho ou operacional (working memory), pelo planejamento e controle inibitório (Fuster, 2002). Em outros termos, a memória operacional possibilita a revivência das representações cerebrais (passado, presente, futuro) que se relacionam com estímulos específicos e permitem a instrumentalização neurológica da pessoa para o processamento de uma ação. Tal processo só é possível mediante a inibição das ações impulsivas ou menos adequadas a determinado contexto, como também a limitação máxima da satisfação imediata na seleção da ação visando uma decisão que gere conseqüências com o maior número possível de vantagens ajustadas e encadeadas ao momento de cada pessoa (Palmini, 2004; Pennington \& Ozonoff, 1996).

Todo este complexo funcionamento cerebral pode ser compreendido dentro da expressão "flexibilidade mental" na resolução de problemas, que designa, em suma, a capacidade de adaptar suas escolhas às contingências e, diante das possíveis eventualidades, mudar de opinião supondo a inibição da primeira escolha em favor de outra (Gil, 2002; Portuguez \& Charcat, 1998).

Os principais aspectos neuropsicológicos avaliados em indivíduos com comprometimento nas funções do córtex pré-frontal são ligados às dificuldades em tarefas que exigem capacidade de planejamento e estabelecimento de estratégias para solução de problemas, além de avaliação e controle do próprio comportamento diante das interferências. Tais dificuldades resultam em falta de flexibilidade diante de situações diferenciadas, precária análise e aproveitamento de sinais ambientais externos para orientar as escolhas e respostas apropriadas. Este funcionamento também pode ser associado à impossibilidade da pessoa de deixar de lado uma forma de compreensão e utilizar ou partir para a busca de outra, fazendo com que persevere numa única estratégia para solucionar os problemas. Tal quadro é complementado pela tendência destes indivíduos de agir no aqui e agora (temporalidade concreta) e pouca preocupação com as conseqüências de suas condutas (Gil, 2002; Portuguez \& Charcat, 1998).

Estudos neuropsiquiátricos demonstram uma associação significativa entre medidas de disfunção executiva através da testagem neuropsicológica e comportamento homicida. Destaca-se o estudo de Sreenivasan et al. (1997) com pacientes que cometeram crimes, dentre eles o homicídio, e encontravam-se internados em hospitais psiquiátricos e penitenciários. Estes estudiosos concluíram que os preditores de recidividade violenta foram a falta de controles morais sobre o comportamento e a inflexibilidade cognitiva, sendo que esta poderia estar mais ligada com o comportamento recidivo do que com o diagnóstico psiquiátrico sozinho e seria consistente com um modelo neuropsicológico de violência que implicasse disfunção pré-frontal.

A conexão entre disfunção frontal e homicídio foi apontada em diversos estudos (Dolan, Deakin, Roberts \& Anderson, 2002; Gatzke-Kopp, Raine, Buscsbaum \& LaCasse, 2001; Laakso et al., 2002) através da avaliação neurológica e neuropsicológica de indivíduos com pro- 
cesso legal por homicídio. Ainda, Jozef, Silva, Greenhalgh, Leite e Ferreira (2000) realizaram estudo sobre disfunção cerebral e psicopatia (transtorno de personalidade antisocial) em homicidas. Apontaram evidências de correlação entre disfunção frontal e comportamento homicida, principalmente nos indivíduos que tinham tendência a cometer homicídios instrumentais, ou seja, aqueles que ocorriam no âmbito de uma criminalidade mais ampla, com menor fator emocional envolvido.

Estes estudos empíricos referidos acima ilustram a relevância do funcionamento pré-frontal para o comportamento violento. No entanto, não foram identificadas, na literatura científica indexada, pesquisas que utilizem amostras com indivíduos que cometeram homicídio qualificado relacionadas com flexibilidade mental. Em geral, a literatura apresenta amostras de homicidas sem a designação legal/criminológica do tipo de homicídio cometido ou que este aspecto seja considerado dentre as variáveis estudadas.

Assim sendo, o presente estudo teve como objetivo principal avaliar a flexibilidade na resolução de problemas em indivíduos que cumprem pena por homicídio qualificado. Verificar também se houve diferença entre a flexibilidade na resolução de problemas em indivíduos que cumprem pena por homicídio qualificado quando comparados com indivíduos da população em geral (sem antecedentes criminais).

\section{Método}

\section{Participantes}

O estudo foi quantitativo de tipo transversal, tendo num primeiro momento um enfoque descritivo e, posteriormente, uma comparação entre variáveis. A amostra constituiuse por conveniência ou não aleatória, por 60 indivíduos, com idade entre 21 e 40 anos. Divididos em dois grupos, um com 30 indivíduos (G1) que cumprem pena (penitenciária masculina) em regime fechado por homicídio qualificado (art. $121 \$ 2^{\circ}$ do CPB), pareado com outro grupo composto por 30 sujeitos (G2) sem antecedentes criminais, da população geral. O tamanho amostral $(n=60)$ foi estabelecido considerando o nível de confiança de $95 \%$ e a margem de erro máxima estimada em 1,5\%.

Os critérios de exclusão da amostra para ambos os grupos foram a realização de eletroconvulsoterapia nos últimos meses; utilização de psicotrópicos com propriedade anticolinérgica ou benzodiazepínica, ou qualquer tipo de medicamento que possa causar alteração na cognição no momento da aplicação dos instrumentos; apresentação de transtorno ou sintomas psicóticos, retardo mental e/ou desordens neurológicas.

Como critérios de inclusão utilizaram-se para o G1 o cumprimento de pena por homicídio qualificado em estabelecimento penal de segurança média e regime fechado, ter no mínimo cinco anos de escolaridade e não apresentar nenhum dos critérios de exclusão. E para o G2 os critérios foram a ausência de histórico de antecedentes criminais, ter no mínimo cinco anos de escolaridade e não apresentar nenhum critério de exclusão descrito. As características quanto a sexo, idade, escolaridade e classe social apresentadas por cada um dos indivíduos do G1, deram origem às características para a organização do grupo de 30 sujeitos sem antecedentes criminais (G2) necessárias para o pareamento.

\section{Instrumentos}

Para a caracterização da amostra foi utilizada uma ficha de dados sócio-demográficos e clínicos. Para avaliar a presença de quadros psicopatológicos empregou-se a MiniInternational Neuropsychiatric Interview (MINI), na versão brasileira 5.0 traduzida por P. Amorim (Sheehan, Janavs, Baker, Sheehans \& Knapp, 2000).

Como medida de avaliação da inteligência geral foram utilizados alguns subtestes da Escala de Inteligência Wechsler para Adultos - WAIS-III (2004) na versão adaptada e padronizada para o Brasil por Elisabeth Nascimento. Esse instrumento compreende duas subescalas: uma verbal e outra de execução, ambas com 7 subtestes. Para a pesquisa atual foi administrado o subteste executivo $\mathrm{Cu}$ bos, que visa avaliar a capacidade de análise e síntese, conceitualização visoespacial, coordenação visomotora espacial e estratégias de solução de problemas. O subteste de execução Código que avalia a coordenação visomotora, a atenção e a memória. Por fim, Vocabulário um subteste verbal que investiga conhecimento semântico, inteligência geral, estimulação do ambiente e antecedentes educacionais. A escolha por estas subescalas deveu-se ao fato de que vários estudos apontam as mesmas como medidas fidedignas de triagem, da presença de déficit cognitivo. Vocabulário é considerado como a melhor medida singular de inteligência geral; Cubos como tendo alta correlação com QI de execução e a melhor medida de "fator g" de toda a escala; e Código por ser bastante valorizado como teste de velocidade, permitindo avaliar a manutenção da atenção e da memória, além da avaliação da flexibilidade mental (Cunha, 2000; Lezak, 1995; WAIS-III, 2004).

Para avaliar flexibilidade na resolução de problemas fezse uso do Teste Wisconsin de Classificação de Cartas e do Teste Stroop de Cores e Palavras. O Teste Wisconsin de Classificação de Cartas - WCST (2005) é composto por O4 cartas-estímulo e 128 cartas-resposta. A tarefa do indivíduo é combinar as cartas-resposta com uma das quatro cartas-estímulo conforme uma das categorias (cor, forma, número, outra). A cada dez acertos sucessivos o critério é mudado sem aviso prévio ao examinando, que apenas recebera a orientação se a colocação atual está correta ou errada. O procedimento se repete até que o individuo complete 6 séries corretamente classificadas ou termine o número de cartas. O levantamento dos dados é realizado estabelecendo escores quanto ao número total de erros (indicativo quanto à tendência geral a responder errado), aos erros perseverativos (quando o sujeito persiste em responder a uma categoria específica que é incorreta); aos erros não perseverativos (o sujeito responde de forma errônea e não as combina com o princípio perseverante ou vigente), e as repostas de nível conceitual (denotam a 
compreensão do sujeito com os princípios corretos de classificação), (Cunha, 2000; Heaton, Chelune, Talley, Kay \& Curtiss, 1993; Lezak, 1995). O teste WCST foi adaptado e validado para a realidade brasileira numa população infantil e de adolescentes por Cunha et al. Já para a população adulta está em processo de validação, devido a isso foram utilizadas como medidas os escores brutos apresentados em cada categoria analisada.

No Teste Stroop de Cores e Palavras a tarefa requer a modificação da atenção em duas dimensões do estímulo (cor e palavra). É constituído de três partes com 100 itens cada. Na primeira, Cor, o indivíduo realiza a leitura oral dos nomes das cores impressas em cor preta e organizadas em fileiras. Na segunda, Palavra, realiza a leitura dos nomes das cores impressas em tintas coloridas correspondentes. Na última parte, Cor e Palavra, faz a nomeação das cores da tinta em que as palavras estão impressas, ignorando a palavra que esta impressa em cada item. Nas três partes o indivíduo é orientado a fazer a leitura o mais rápido possível. Os escores são atribuídos mediante o número de itens lidos em cada parte, num tempo estipulado de 45 segundos. Como o instrumento ainda não se encontra validado para a população brasileira, foram utilizados nas três partes os escores brutos seguidos pelo teste de diferença entre médias, além da média dos escores brutos na análise dos dados (Lezak, 1995; Spreen \& Strauss, 1998).

\section{Procedimentos}

Este estudo foi desenvolvido em uma penitenciária de segurança média da região metropolitana de Porto Alegre, após a autorização da Direção do estabelecimento. Os sujeitos foram contatados individualmente durante seu cumprimento de pena, quando então foi conversado sobre o estudo e esclarecido seu objetivo e principais pontos. Após a concordância em participar da pesquisa, foi entregue o Termo de Consentimento Livre Esclarecido, assinado em duas vias. Foram realizados dois encontros (dentro do recinto prisional, na sala de atendimento psicológico) de aproximadamente 1 hora cada, para a administração dos instrumentos, com um intervalo de no máximo cinco dias entre cada encontro. Os sujeitos sem antecedentes criminais, da população geral, foram contatados individualmente, por conveniência, de acordo com as características necessárias para o pareamento. A aplicação dos instrumentos também foi realizada em dois encontros, de aproximadamente 1 hora cada, em local previamente estabelecido pela pesquisadora e em concordância com o sujeito.

Os dados coletados foram processados e analisados estatisticamente através de técnicas descritivas (média, distribuição de freqüências). Para amostras independentes foi utilizado o teste Mann-Whitney e Student. O nível de significância estatístico adotado foi de $5 \%$.

O projeto deste estudo foi avaliado e aprovado pelo Comitê de Ética e Pesquisa da Pontifícia Universidade Católica do Rio Grande do Sul - PUCRS.

\section{Resultados}

Dos 60 sujeitos que constituíram a amostra, 30 cumpriam pena em regime fechado por homicídio qualificado e configuraram o G1 nas seguintes características: sexo masculino, com idade média atual de 28,77 anos $(D P=$ 5,95). A idade média na ocasião do crime foi de 23,37 anos, com variação de 18 a 38 anos. Em relação à classe social, $24(80 \%)$ sujeitos eram de classe baixa e $6(20 \%)$ eram de classe média. Quanto ao grau de escolaridade, 24 (80\%) haviam cursado o Ensino Fundamental Incompleto, 2 $(6,7 \%)$ o Fundamental Completo, 3 (10\%) Ensino Médio Incompleto, 1 (3,3\%) Ensino Médio Completo. Em decorrência do pareamento, o G2 apresenta exatamente as mesmas características sócio-demográficas descritas.

Quanto à configuração/moradia familiar, à época do crime, 18 sujeitos residiam com suas esposas/companheiras e filhos, 6 residiam com os pais, 2 com irmãos e 4 moravam sozinhos. Quanto aos antecedentes criminais familiares dos homicidas, foi identificado que 53,33\% desses sujeitos tinham parentes (especialmente pais, irmãos, tios) que já haviam cometido crimes (na maior parte assaltos à mão armada e homicídios) em data anterior a época do homicídio qualificado.

Dos 30 sujeitos que cometeram homicídio qualificado, $20(66,7 \%)$ eram primários e $10(33,3 \%)$ já haviam cumprido pena ou estavam cumprindo outras penas (furto, assalto à mão armada, latrocínio, lesões corporais, estupro) concomitantes com o homicídio qualificado atual. Destes últimos, 2 sujeitos já tinham cumprido pena por tentativa de homicídio, 1 pela autoria de dois homicídios e outro sujeito cumpria pena por dois homicídios qualificados na época do estudo.

As vítimas do homicídio atual eram conhecidas dos sujeitos (agressores) em 66,7\% dos casos, companheira em $10 \%$, desconhecidos em 10\%, amigo e parente com 6,6\%, cada um. Quanto ao sexo das vítimas, 3 (10\%) eram do sexo feminino e 27 (90\%) eram do sexo masculino. O motivo do homicídio foi $35,5 \%$ por desentendimento banal com a vítima, $13 \%$ por domínio territorial pela venda de drogas, $13 \%$ financeiros, $9,7 \%$ rixa, 9,7\% amoroso, $6,4 \%$ vingança, $6,4 \%$ familiar, recompensa e fuga da polícia cada um $\operatorname{com} 3,2 \%$.

Como métodos empregados para o homicídio atual, 25 sujeitos $(83,3 \%)$ utilizaram para matar a arma de fogo, 2 (6,7\%) a agressão física (socos e pontapés), 2 (6,7\%) objeto pérfuro-cortante (faca e espeto) e 1 (3,3\%) incêndio (ateou fogo à residência da vítima).

Através da Mini-International Neuropychiatric Interview (MINI), pode-se verificar no G1 (homicidas) que dos 30 sujeitos, 16 (53,3\%) não atenderam aos critérios para diagnóstico psicopatológico. Dos 14 (46,7\%) sujeitos que atenderam aos critérios, 6 apresentaram só um diagnóstico, enquanto os $8(26,7 \%)$ restantes tinham diagnóstico com comorbidades. Dentre os 6 sujeitos que atenderam critérios só para um diagnóstico, 3 apresentavam Transtorno de Personalidade Anti-social, 2 Transtorno 
de Ansiedade Generalizada e 1 Episódio Depressivo Maior Recorrente. Os 8 sujeitos que atenderam critérios para mais de um diagnóstico, tiveram como diagnóstico principal Episódio Depressivo Maior (50\%), Transtorno de Personalidade Anti-social (37,5\%) e Transtorno de Ansiedade Generalizada (12,5\%), como comorbidades: Abuso de Substância, Episódio Hipomaníaco Passado, Ataques pobres em sintomas de pânico, Transtorno de Personalidade Anti-social e Risco Suicídio Baixo.

Quanto aos dados da configuração/moradia familiar do grupo sem antecedentes criminais (G2), constatou-se que $43,3 \%$ residem com sua esposa/filhos, $40 \%$ com os pais, $10 \%$ com parentes, $3,3 \%$ com o amigo e $3,3 \%$ morava sozinho. Neste grupo, $4(13,3 \%)$ sujeitos tinham um familiar (primos e tio) que cometeu delito do tipo furto (50\%) e assal to (50\%), não envolvendo vítimas fatais ou lesões corporais.

No G2, 26 (86,7\%) não atenderam aos critérios para diagnóstico psicopatológico, e dos 4 (13,3\%) sujeitos que atenderam critério, 3 (10\%) apresentaram só um diagnóstico. Dentre estes 3 sujeitos, 2 apresentaram Transtorno Distímico e 1 Transtorno de Pânico a Vida Inteira. Um sujeito preencheu critérios para diagnóstico principal de Dependência Álcool com comorbidade Transtorno Ansiedade Generalizada.

Como medida de inteligência geral, realizou-se o cálculo das médias a partir de escores ponderados em G1 e G2, em cada um dos subtestes do WAIS-III (Cubos, Vocabulário e Código). O G1 apresentou em Cubos $M=9.80$, em Vocabulário $M=10.40$, e em Código $M=9.07$. Enquanto que o G2 em Cubos apresentou $M=11.13$, em Vocabulário $M=12.40$ e em Código $M=10.70$. Tais resultados indicam que os sujeitos da amostra apresentam desempenho intelectual dentro do esperado para sua idade. Ainda cabe mencionar que foram encontradas diferenças significativas entre as médias dos dois grupos em Cubos ( $\mathrm{t}=-$ 3,297; $p=0,002)$, Códigos ( $\mathrm{t}=-4,913 ; p<0,001)$ e Vocabulário $(\mathrm{t}=-3,684 ; p=0,001)$.

$\mathrm{Na}$ Tabela 1 e 2 , apresentam-se os resultados das médias obtidas nos instrumentos para avaliação da flexibilidade na resolução de problemas. Nestas tabelas, verifica-se que G1 nas categorias do WCST: erros perseverativos $(M=36,33, D P=22,71)$, erros não perseverativos $(23,46$, $D P=13,96)$, total de erros $(M=59,80, D P=22,09)$ e respostas de nível conceitual $(M=38,60, D P=20,99)$, bem como, nas categorias do Teste Stroop: Palavras $(M=82,53$, $D P=10,72)$, Cores $(M=62,23, D P=9,95)$ e Cores e Palavras $(M=39,10, D P=11,97)$, apresentou escores inferiores ao $\mathrm{G} 2$, com diferença estatisticamente significativa. Estes dados indicam que os sujeitos que cumprem pena por homicídio qualificado (G1) apresentam menor flexibilidade na resolução de problemas quando comparados com indivíduos sem antecedentes criminais (G2).

Tabela 1

Médias e Desvios-Padrão nas Categorias do Teste WCST, em ambos os Grupos $(n=60)$

\begin{tabular}{lllllll}
\hline Categorias do instrumento & \multicolumn{2}{c}{$\begin{array}{c}\text { Grupo 1 } \\
\text { (Homicidas) }\end{array}$} & DP & Grupo 2 & & \\
\cline { 2 - 7 } & $M$ & População Geral) & Mann-Whitney & $p$ \\
\hline Total de erros & 59,80 & 22,09 & 29,00 & 12,87 & $-5,18$ & $<0,001$ \\
Erros perseverativos & 36,33 & 22,71 & 15,40 & 7,04 & $-4,84$ & $<0,001$ \\
Erros não perseverativos & 23,46 & 13,96 & 13,60 & 6,76 & $-3,66$ & $<0,001$ \\
Respostas nível conceitual & 38,60 & 20,99 & 67,74 & 11,24 & $-5,26$ & $<0,001$ \\
\hline
\end{tabular}

Tabela 2

Médias e Desvios-Padrão nas Categorias do Teste Stroop, em ambos os Grupos $(n=60)$

\begin{tabular}{|c|c|c|c|c|c|c|}
\hline \multirow[t]{2}{*}{ Categorias do instrumento } & \multicolumn{2}{|c|}{$\begin{array}{r}\text { Grupo } 1 \\
\text { (Homicidas) }\end{array}$} & \multicolumn{2}{|c|}{$\begin{array}{c}\text { Grupo } 2 \\
\text { (População Geral) }\end{array}$} & \multirow[b]{2}{*}{ Student } & \multirow[b]{2}{*}{$p$} \\
\hline & $M$ & $D P$ & $M$ & $D P$ & & \\
\hline Palavras & 82,53 & 10,72 & 93,13 & 10,31 & $-3,903$ & $<0,001$ \\
\hline Cores & 62,23 & 9,95 & 68,20 & 10,90 & $-2,215$ & 0,031 \\
\hline Cores e Palavras & 39,10 & 11,97 & 44,37 & 8,411 & $-1,992$ & 0,050 \\
\hline
\end{tabular}




\section{Discussão}

Estabelecendo um paralelo com o perfil dos sujeitos homicidas traçado em outros estudos (CLAVES, 2001, 2002; Krug, Dahlberg, Zwi \& Lozano, 2003), foi possível verificar que o G1 também se constituiu por sujeitos com baixos recursos financeiros/econômicos e com escassa educação formal em termos de anos de estudo e utilizando, como principal método de assassinato, a arma de fogo.

A idade média de 23,37 anos na ocasião do crime coincide com o que é narrado na literatura, como também indica uma tendência mundial de aumento dos homicídios cometidos por jovens (Josef, 1997; Krug et al., 2003). Além disso Nestor (1992) e Sreenivasan et al. (1997) postularam em suas pesquisas com populações criminosas, que um dos fatores de risco associado à periculosidade seria $\mathrm{o}$ comportamento criminoso passado. Assim é importante destacar que 33,3\% dos indivíduos do G1 eram jovens e já haviam sido condenados por outros crimes, sendo que alguns destes reincidiram em homicídios consumados ou tentados.

Os padrões familiares criminosos também aparecem associados ao comportamento violento (Dalberg, 1998). No G1 se percebe que mais da metade dos sujeitos possuem parentes em primeiro grau que foram co-autores em homicídio qualificado ou já haviam cometido crimes em sua maioria violentos, o que certamente os deixa vulneráveis, através da convivência com modelos familiares criminosos. Cabe destacar que não é totalmente possível inferir a direção causal nesta correlação, no sentido de que se ter familiares criminosos vem antes ou depois de se cometer um homicídio.

Por outro lado, o perfil das vítimas aponta a sobremortalidade masculina de 9:1, principalmente de indivíduos considerados "conhecidos" (aqui entendidos como aquelas pessoas de convivência social mais ou menos constante e sem maiores laços afetivos com o agressor). As vítimas femininas são oriundas de desentendimentos amorosos ou conjugais e suas mortes, causadas pelo esposo ou companheiro, estão predominantemente ligadas à vivência de perda da pessoa "amada" (vítima). Tal atuação criminosa aparece, de acordo com descrito por Bifano, Boechat, Gauer e Machado (2003) como uma defesa e antecipação frente a esta "perda". Tais dados confirmam a afirmação de que o homicídio tende a ser freqüentemente um crime intrafamiliar, entre amigos, conhecidos, ou seja, dentro de uma rede social próxima (Jozef, 1997; Krug et al., 2003).

Em acordo com a definição de homicídio qualificado pelo Código Penal Brasileiro, a prevalência dos desentendimentos banais com a vítima $(35,5 \%)$ nos casos investigados neste estudo, confirma a classificação e a reprovabilidade do crime diante do motivo fútil. Dentre os motivos que levaram os sujeitos do G1 a cometerem homicídio, também é possível destacar a ação intencional do agressor em produzir a morte do outro, baseando-se nesta intenção como solução para um conflito momentâneo ou para obter um ganho secundário (financeiro, domínio territorial, vingança).

Diante desta configuração, a presença de uma arma de fogo sem dúvida altera o contexto de uma confrontação violenta, ou até numa discussão banal, de forma radical. É possível que a presença da arma de fogo, intrinsecamente, possa provocar violência, como postulou Berkowitz (1989), ou então canalizar uma descarga agressiva que, sem sua presença, certamente não teria resultado fatal. Por outro lado, a utilização da arma de fogo como método preponderante nos homicídios parece uma escolha "simples" diante do acesso facilitado a este artefato, da sua potencialidade fatal, da sua inserção cada vez maior num contexto da criminalidade mais ampla (por exemplo, latrocínio e tráfico), do uso indiscriminado pela população em geral como instrumento para a defesa. Neste sentido, a arma usada para se defender também acaba por matar, revelando um paradoxo nas diversas sociedades que ainda precisam deliberar sobre assuntos polêmicos como porte de armas, formas de penalização, a indústria bélica, entre outros.

No tocante à história psiquiátrica dos sujeitos investigados, não há referências de internações psiquiátricas. No entanto, uma parcela razoável de sujeitos do G1 atendeu aos critérios para diagnóstico psicopatológico $(46,7 \%)$, podendo tal fator contribuir para o risco de comportamento homicida, como apontado na literatura (Asnis, Kaplan, Hundorfean \& Saeed, 1997; Jozef \& Silva, 2002). Valendo-se da explicação de Swanson (1994), entende-se que esta possível relação entre homicídio e transtorno psiquiátrico, encontrado no G1, deva-se mais à sintomatologia psiquiátrica ativa do que à história de hospitalização psiquiátrica, a qual mostrou-se ausente no grupo.

Considerando os aspectos mencionados, pode-se inferir que os sujeitos homicidas, neste estudo, mostram-se vulneráveis em termos sócio-econômicos, educacionais, criminalidade familiar e em distúrbios psiquiátricos. Estes componentes são amplamente apontados como fatores de risco para o desencadeamento da criminalidade (Dornelles, 1988; Gauer \& Guilhermano, 2001; Minayo \& Souza, 1998; Walker, 2001). Com o pareamento necessário para a organização do G2 quanto às características sexo, idade, escolaridade e classe social, os dois grupos (G1 e G2) possuem os mesmos dados sócio-demográficos e ainda alguns sujeitos do G2 também apresentam transtornos psiquiátricos e histórico criminal na família. Assim, estes resul tados mostram que estes fatores, tidos como de risco para o comportamento homicida, não podem ser considerados os únicos ou os mais preponderantes para o homicídio, pois com características similares, os sujeitos do G2 não incidiram em conduta criminosa. Pode-se inferir que por algum(s) outro(s) motivo(s), estes indivíduos do G2 acharam outras formas de lidar ou solucionar as possíveis adversidades da vida que não de maneira violenta e fatal que os sujeitos do G1. 
Deste modo, outros componentes, certamente, têm um peso maior para influenciar o comportamento homicida, entre eles estaria o funcionamento cognitivo, mais especificamente a flexibilidade para resolver problemas, como apontado por Blake et al. (1995), Dolan et al. (2002), Sreenivasan et al. (1997) entre outros autores.

Assim, constatou-se que os indivíduos que cometeram homicídio qualificado (G1) apresentam pior desempenho no Teste Wisconsin de Classificação de Cartas e o no Teste Stroop, evidenciando uma deficiência na flexibilidade cognitiva e, conseqüentemente, um possível indicativo de disfunção pré-frontal (o que necessitaria de maiores investigações), como foi encontrado em estudos com homicidas por Blake et al. (1995), Jozef et al. (2000), Raine et al. (1997), e Sreenivasan et al. (1997).

Através dos escores alcançados nas categorias do WCST pelo G1, quanto ao número elevado do total de erros, de erros perseverativos e não perseverativos, além do menor número de repostas de nível conceitual, constata-se um prejuízo no desempenho dos homicidas quando comparados ao G2. Ao considerar estes dados, pode-se inferir que frente à necessidade de analisar uma diversidade de estímulos para fazer sua escolha diante de uma questão, os indivíduos que cometeram homicídio qualificado parecem ter dificuldade para identificar as estratégias mais adequadas para encontrar a solução deste problema, não conseguindo valer-se da aprendizagem anteriormente armazenada na memória operacional no que diz respeito às experiências negativas ou positivas obtidas em situações/ emoções similares àquele momento. Mesmo com a sinalização externa indicando que determinada resposta não é correta (no caso do pesquisador no momento da aplicação do instrumento) os sujeitos do G1 demonstraram dificuldade em alterar seu estilo de resposta imprópria e de inibir esta tendência comportamental mais imediata em prol de uma resposta mais adequada e elaborada. Tal processo pode também servir como padrão de respostas destes sujeitos nas situações adversas da vida diária.

Ainda, estudos apontam que os homicidas apresentam menor habilidade em mobilizar estratégias comportamentais quando há estímulos competindo (Blake et al., 1995). Este dado é comprovado na amostra através do desempenho inferior dos indivíduos do G1 no Teste Stroop e, em concomitância ao verificado no WCST, em forma de erros perseverativos altos. Estes resultados evidenciam que estabelecida uma estratégia de resolução, os indivíduos do G1 tendem a manter-se num princípio perseverativo, o qual limita suas percepções para outros modos de resolução mais eficientes e, mesmo com a indicação de erro, permanecem no mesmo padrão de respostas inadequadas.

Autores como Fuster (2002) e Palmini (2004) descrevem a importância da memória neste complexo funcioamento cerebral compreendido como flexibilidade na resolução de problemas. Através da memória são revividas as representações mentais de emoções e experiências (presentes, passadas, futuras) e que se relacionam a estímulos específicos do momento, permitindo toda a preparação mental para a escolha e o processamento das ações envolvidas para tal. Assim, a memória instrumentaliza o indivíduo sobre o quão boas ou ruins foram as vivências para então decidir se segue na mesma linha de resolução ou não.

Por outro lado, os sujeitos que cometeram homicídio qualificado apresentaram escores mais baixos ao do G2 no subteste Códigos (WAIS- III), dado que pode servir como algum indício da possível presença de prejuízos na memória, já que este subteste avalia a memória psico-motora, a qual requer certas condições tanto para aprendizagens novas como para atividades continuadas (Cunha, 2000). Qualquer nível de dificuldade no processamento da memória poderia prejudicar os indivíduos homicidas no resgate das vivências passadas, fazendo-os vivenciar cada problema como se fosse a primeira vez a ser resolvido, incrementando maior impulsividade e ansiedade ao empenho na solução da conflitiva específica.

Através das elucidações de Palmini (2004) e Pennington e Ozonoff (1996), entende-se que a integração dos diversos fatores que envolvem o processo para a tomada de decisão parece estar afetada nos indivíduos homicidas deste estudo, considerando-se também as possíveis falhas na utilização de sinais somáticos ou emocionais para guiar suas condutas. Ou seja, a representação de quão bons ou ruins foram os resultados de sua ação dificilmente são acessados ou fazem parte de suas deliberações adotadas. Assim, a dificuldade em avaliar e controlar o próprio comportamento resultaria em falta de flexibilidade diante das situações diferenciadas, levando a perseveração de uma única estratégia de solução. Associado a isso, quando se verifica o grande número de erros (perseverativos e não perseverativos) do G1, entende-se que uma vez que os indivíduos homicidas definem o seu modo de solução dos problemas, este padrão dificilmente modifica, mesmo quando pontuado como uma estratégia errônea - o que caracterizaria por menor habilidade cognitiva para resolver problemas.

A partir deste contexto considera-se que, nos indivíduos do G1, a falta de flexibilidade cognitiva na escolha de soluções mais adequadas aos problemas específicos que, em dado momento envolveram um alvo humano (as vítimas), pode ter estado associada, entre outros fatores possivelmente implicados na ocasião do delito, ao comportamento intencional de matar. Sendo assim, concluise que os indivíduos que cumprem pena por homicídio qualificado, aqui investigados, apresentaram níveis diferenciados de flexibilidade metal, resultando em uma menor habilidade cognitiva na resolução dos problemas.

\section{Considerações Finais}

Com base nos resultados alcançados, pode-se constatar neste estudo e com esta amostra, que os indivíduos que cumprem pena por homicídio qualificado apresentam menor flexibilidade na resolução de problemas do que os indivíduos sem antecedentes criminais, podendo este ser um fator de peso para o comportamento homicida. 
Este estudo levou em consideração a avaliação neuropsicológica em termos de flexibilidade cognitiva dos sujeitos no momento presente da pesquisa. Sem dúvida, tornam-se necessários novos estudos com uma amostra maior e outras variáveis de controle, tais como os diversos fatores envolvidos no encarceramento destas populações, com sujeitos do sexo masculino e feminino que cometeram homicídio qualificado e de classes sociais diferentes, com o objetivo de ampliar os resultados aqui alcançados.

\section{Referências}

Asnis, G. M., Kaplan, M. L., Hundorfean, G., \& Saeed, W. (1997). Violence and homicidal behaviors in psychiatric disorders. Psychiatric Clinics of North America, 20(2), 405-25.

Berkowitz, L. (1989). Frustration - Aggression hypothesis: Examination and reformulation. Psychological Bulletin, 106, 5973 .

Bifano, A. H., Boechat, L. F., Gauer, G. J. C., \& Machado, D. S. (2003). Vulnerabilidade do Feminino: Homicídio contra a esposa. In G. J. C. Gauer \& D. S. Machado (Eds.), Filhos e vítimas do tempo da violência (pp. 71-82). Curitiba, PR: Juruá.

Blake, P.Y., Pincus J. M., \& Buckner C. (1995). Neurologic abnormalities in murderers. Neurology, 45, 1641-7.

Centro Latino-americano de Estudos de Violência e Saúde Jorge Careli. (2001). Perfil de mortalidade por causas externas no Brasil: Uma análise temporal das décadas de 80 e 90. Rio de Janeiro, RJ: Autor. Retirado em jun. 2003, de http:// CLAVES/ CENEPI.org.gov.br

Centro Latino-americano de Estudos de Violência e Saúde Jorge Careli. (2002). Padrão de mortalidade por homicídios no Brasil, 1980 a 2000. Rio de Janeiro, RJ: Autor. Retirado em jun. 2003, de http:// CLAVES/CENEPI.org.gov.br

Cunha, J. A. (2000). Psicodiagnóstico: Vol. 5 (5. ed). Porto Alegre, RS: Artes Médicas.

Dalberg, L. (1998). Youth violence in the United Stares: Major trends, risk factors and prevention approaches. American journal of Preventive Medicine, 14, 259-272.

Dolan, C. M., Deakin, J. F. W., Roberts, N., \& Anderson, I. M. (2002). Quantitative frontal and temporal structural MRI studies in personality-disordered offenders and control subjects. Psychiatry Research: Neuroimaging, 116, 133-149.

Dornelles, J. R. W. (1988). Criminologia: Introdução a seus fundamentos teóricos. São Paulo, SP: Brasiliense.

Escala de Inteligência Wechsler para Adultos. (2004). WAIS: Manual (E. Nascimento, Adaptação e padronização brasileira). São Paulo, SP: Casa do Psicólogo.

Filley C. M., Price, B. H., Nell, V., Antoinette, T., \& Morgan, A. S. (2001). Toward and Understanding of violence: Neurobehavioral aspects of unwarranted physical aggression Aspen Neurobehavioral Conference Consensus Statement. Neuropsychiatry, Neuropsychology and Behavior Neurology, 14.(1), 1-14

Foster, H. G., Hillbrand, M., \& Silverstein, M. (1993). Neuropsychological deficit and aggressive behavior: A prospective study. Progress in Neuro-Psychopharmacology \& Biological Psychiatry, 17, 939-946.

Fuster, J. M. (2002). Frontal lobe and cognitive development. Journal of Neurocytology, 31, 373-385.
Gatzke-Kopp, L. M., Raine, A., Buscsbaum, M., \& LaCasse, L. (2001). Temporal lobe deficits in murderers: EEG findings undetected by PET. The Journal of Neuropsychiatry and Clinical Neurosciences, 13(4), 486-491.

Gauer, G. J. C., \& Guilhermano, T. F. (2001). Fatores biológicos associados à conduta agressiva. In G. J. C. Gauer (Ed.), Agressividade - Uma leitura biopsicossocial (pp. 11-33). Curitiba, PR: Juruá.

Giancola, P. R., \& Zeichner, A.(1994). Neuropsychological performance on tests of frontal-lobe functioning and aggressive behavior in men. Journal of Abnormal Psychology, 103(4), 832-835.

Gil, R. (2002). Neuropsicologia (2. ed). São Paulo, SP: Santos.

Heaton, R. K., Chelune, G. J., Talley, J. L., Kay, G. G., \& Curtiss, G. (1993).Wisconsin Card Storting Test-WCST. Manual. Jacksonville, FL: Psychological Assessment Resources.

Jozef, F. (1997). O criminoso homicida: Estudo clínico-psiquiátrico. Tese de Doutorado não-publicada, Curso de Pós-Graduação em Psiquiatria, Universidade Federal do Rio de Janeiro, RJ.

Jozef, F., \& Silva, J. A. R. (2002). Homicídio e doença mental. Psiquiatria a Prática Médica, 34(4). Retirado em 05 maio 2003, de http://www.unifesp.br/dpsiq/polbr/ppm/original 7 _o2.htm

Jozef, F., Silva, J. A. R., Greenhalgh, S., Leite, M. E. D., \& Ferreira, V. H. (2000). Comportamento violento e disfunção cerebral: Estudo de homicidas no Rio de Janeiro. Revista Brasileira de Psiquiatria, 22(3), 124-9.

Krug, E. T., Dahlberg, J., Zwi, A. B., \& Lozano, R. (2003). Informe mundial sobre la violencia y la salud. Washington, DC: Publicação Científica y Técnica.

Laakso, M. P., Gunning-Dixon, F., Vaurio, O., Repo-Tiihonen, E., Soininen, H., \& Tiihonen, J. (2002). Prefrontal volumes in habitually violent subjects with antisocial personality disorder and type 2 alcoholism. Psychiatry Research Neuroimaging, 114, 95-102.

Lezak, M. D.(1995). Neuropsychological assessment (3. ed). New York: Oxford.

Minayo, M. C. S., \& Souza, E. R.(1998). Violência e saúde como um campo interdisciplinar e de ação coletiva. História, Ciências, Saúde-Manguinhos, 4(3), 513-531

Nestor, P. G. (1992). Neuropsychological and clinical correlates of murder and other forms of extreme violence in a forensic psychiatric population. The Journal of Nervous and Mental Disease, 180, 418-423.

Oliveira-Souza, R., Ignácio, F. A., Cunha, F. C., Oliveira, D. G., \& Moll, J. (2001). Contribuição a neuropsicologia do comportamento executivo. Arquivos de neuropsiquiatria, 5(3), 526-531.

Palmini, A. (2004). O cérebro e a tomada de decisões. In P. Knapp (Ed.), Teoria cognitivo-comportamental na prática psiquiátrica (pp. 71-88). Porto Alegre, RS: Artes Médicas.

Pedroso, F. A. (1995). Homicídio, participação em suicídio, infanticídio e aborto. Rio de Janeiro, RJ: Aide.

Pennington, B. F., \& Ozonoff, S. (1996). Executive functions and psychopathology development. Journal of Child Psychol. Psychiatric and Allied Disciplines, 37(1), 51-87.

Portuguez, M. W., \& Charcat, H. (1998). Avaliação neuropsicológica do lobo frontal. In J. C. Costa (Ed.), Fundamentos neurobiológicos das epilepsias: Aspectos clínicos e cirúrgicos (pp. 957-973). São Paulo, SP: Lemos.

Raine, A., Buchsbaum, M., \& LaCasse, L. (1997). Brain abnormalities in murderers indicated by positron emission tomography. Biological Psychiatry, 42, 495-508. 
Sheehan, J., Janavs, R., Baker, K. H., Sheehans, E., \& Knapp, M. (2000). Mini- international neuropsychiatric interview (Versão 5.0). Sarasota, FL: University of South Florida.

Spreen, O., \& Strauss, E. (1998) A compendium of neuropsychological testes: Administration, norms and commentary (2. ed). New York: Oxford University Press

Sreenivasan, S., Kirkish, P., Eth, S., Mintz, J., Hwang, S., \& Van Gorp, W. (1997) Predictors of recidivistic violence in criminally insane and civilly committed psychiatric impatiens. International Journal Law Psychiatry, 20(2), 279-91.

Swanson, J. W. (1994). Mental disorder, substance abuse and community violence: An epidemiological approach. In J. Monahan \& H. J. Steadman (Eds.), Violence and mental disorder (pp. 120-137). Chicago, IL: University Chicago Press.

Teste Wisconsin de Classificação de Cartas. (2005). WCST: Manual (J. A. Cunha, C. M. Trentini, I. L. Argimon, M. S. Oliveira, B. S. G. Werlang \& R. G. Prieb, Adaptação e padronização brasileira). São Paulo, SP: Casa do Psicólogo.

Walker, P. L. (2001). A bioarcheological perspective on the history of violence. Annual Review of Anthropology, 30, 573596.

World Health Organization. (1996). Violence: A public health priority (Documento WHO/EHA/SPI.POA. $\mathrm{n}^{\mathbf{0}} 2$ ). Genebra, Switzerland: Author 